\title{
Main Principles of Electromagnetic Pulse Immunity Test Methods for Power System Electronics
}

\author{
Vladimir Gurevich, Ph.D
}

\begin{abstract}
Since the devastating effect of EMP on electronics in the military field has been known for a long time, all military systems are equipped with efficient protection against the impact of EMP. However, EMP is equally dangerous for all civil electronics used in almost every section of today's most important infrastructure of any country, for instance the power industry. These days, the protection of power system electronics against EMP that is capable of disturbing its normal operation or even to completely disable it, becomes extremely important. This article explains special aspects related to testing power system electronics resilience to HEMP and discusses the selection of a proper target, methods, and test pulse parameters.
\end{abstract}

Keywords: High Altitude Electromagnetic Pulse, EMP, electromagnetic compatibility, EMP simulator, HEMP resilience tests.

\section{INTRODUCTION}

The ability of the equipment to operate efficiently under the external electromagnetic interferences is known as Electromagnetic Compatibility (EMC). The procedure for testing equipment for EMC is well proven and described in numerous standards. While EMP (HEMP), or rather its component E1, is just one form of electromagnetic interferences disturbing the power system electronics operation [1, 2], it has several significant differences and features dictating the need for improvement and adjustment of known EMC test procedures.

There are dozens of reports and standards on EMP aspects [3], but only a few dedicate to the procedure for testing the equipment resilience to such an impact [4, 5]. As these tests are highly sophisticated, and power-engineering specialists have no experience in performing them in the field, two publications mentioned above do not give enough basis to effectively test the power system electronics. Several sporadic tests, such as testing Digital Protective Relay SEL-311, can hardly be used as the proper method due to the numerous errors, both in the test procedure and the test pulse parameters selection [6].

Therefore, it is necessary to detail the features of EMP resilience test methods for power system electronics.

\section{FEATURES OF HEMP}

HEMP has the following features that are needed to be considered upon the development of the test procedure. The disturbance has a very short time length (single pulse lasts for several nanoseconds), therefore the failure of the equipment under test (EUT) must be registered within this period. This severely limits the number of EUT modes of operation to be controlled during the test. For example, during the HEMP immunity test it is not possible to change the EUT modes of operation using the PC connected to the EUT in order to observe the EUT response to these changes (as is possible during the normal EMC test in the so-called anechoic chamber, where the EUT is exposed to electromagnetic radiation for a long period).

There is a danger of appearance of the so-called soft faults, soft failures or soft errors, especially in electronics memory elements, which are difficult to discover instantly during the tests. The soft faults may show-up in the tested apparatus only after a long period, for example upon the access to the destroyed memory cells during certain operations, or to the certain program modules. 

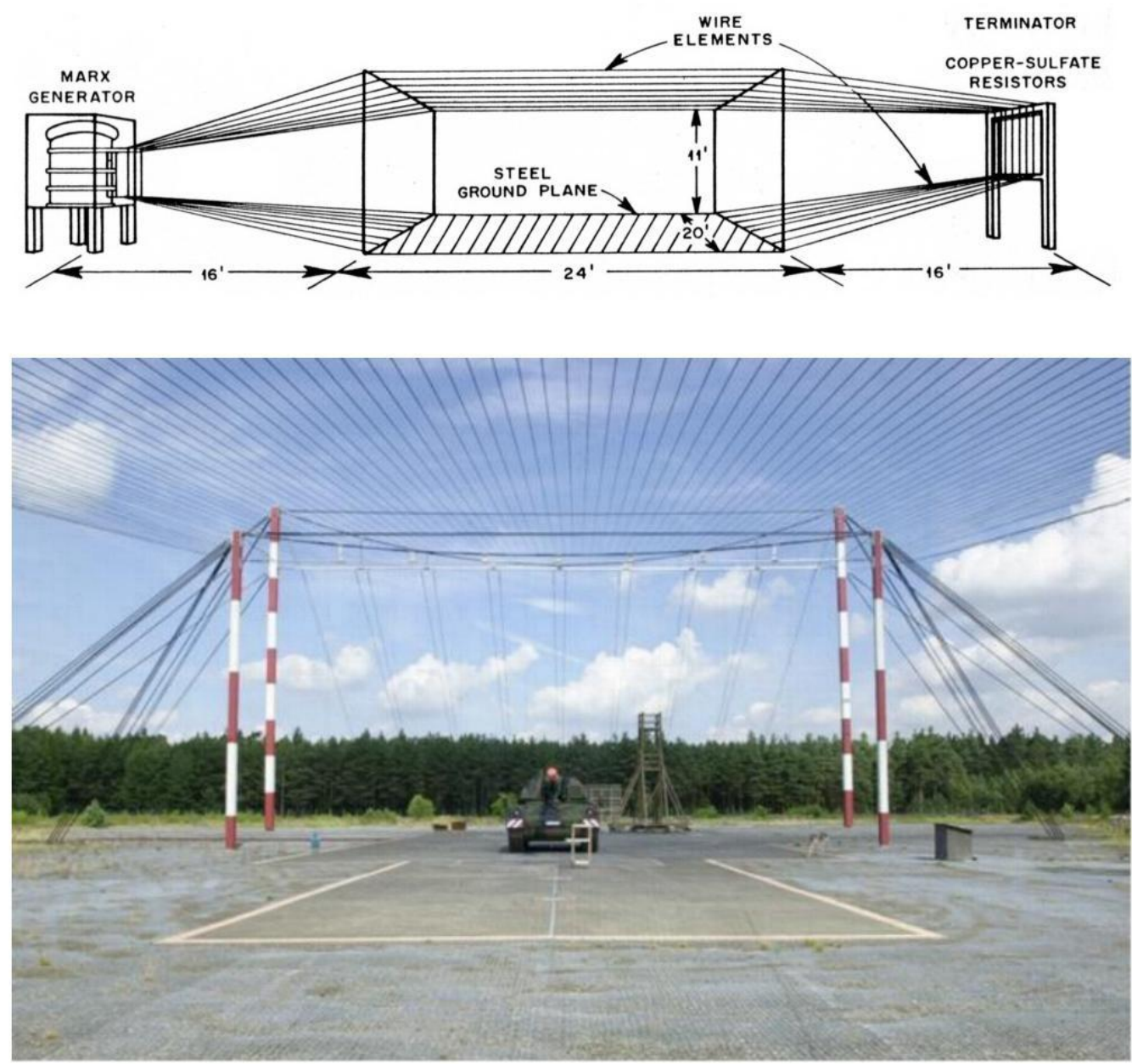

Fig1. Design and appearance of HEMP simulator.

HEMP simulators usually consist of the concrete base with a bonded-in metal mesh acting as the first electrode, and the overhead metal mesh located 10-15 meters above the concrete base, acting as the second electrode. A high-voltage pulse sent from the output of the special generator (usually, a Marx generator built on the set of high-voltage capacitors and triggered spark gaps) is applied between these two electrodes. Generally, the bonded-in mesh connected to the generator cannot be used as the EUT grounding system.

These tests are expensive due to the limited number of test centers in the country and their affiliation to the Ministry of Defense.

It is difficult to select the right EUT configuration, unlike the regular electromagnetic interferences, the HEMP has a rather global nature than a local nature and affects both EUT and its feeding system, grounding system and channels of communication with other facilities. Therefore, instead of a single device, unit or module, a whole system of interconnected devices, units and modules, simulating the real-life environment, including a set of different and distanced grounding points, needs to be tested.

\section{TeST OBJECTIVE}

Due to the complexity and the high cost of the HEMP resilience tests, these tests should be applied only to a limited range of equipment types considered as critically important devices, the lack of which makes even partial operation of electric energy facilities impossible. The selection of the equipment should be the first stage of the test plan development. The second stage should include the clear and transparent description of the test objective, as it will define both the test object and the test procedure. Potential test objectives can be defined as follows:

a. Test the resilience of operating equipment to the highest possible HEMP level without any protection. The objective of this test is to discover elements, units and systems exposed to the HEMP, and thus requiring the protection. 
b. Test the effectiveness of the equipment protection against the maximum possible HEMP level using the minimum set of preinstalled protective means designed for operating a power system. These tests can be use to check the performance of the minimum set of protective means and to discover the types of equipment failures expected under the HEMP.

c. Test the effectiveness of the equipment protection against the maximum possible HEMP level using the complete set of preinstalled protective means designed for newly constructed power systems. This test allows confirming the effectiveness of the most complex and expensive type of protection and justifying the costs of a protection system.

d. Test the operating equipment without special protective means under the series of pulses with the amplitude gradually increasing from $20 \%$ to $100 \%$ of the maximum possible level. The objective of this test is to: 1) find the equipment (or equipment types) mostly exposed to HEMP; 2) define the maximum HEMP amplitude that the unprotected equipment can safely withstand, in order to calculate the needed level of additional protection to improve the equipment's own attenuation to the maximum level provided by the standard.

\section{Features of the test Procedure}

As mentioned above, the number of controllable EUT operation modes during the test is very limited. Thus, these modes can include unauthorized appearance or disappearance of signals at outputs of at least two EUTs interconnected via communication channels in the standby mode (waiting mode), or to high data exchange mode (e.g. in emergency mode). In the latter case, the simulation of the emergency mode start should be synchronized with the initiation of the HEMP pulse.

After the test, because the complex microprocessor-based electronics may have unevident soft failures (even if no appeared damages or failures are registered during the test or the inspection), the equipment should further undergo a complete and thorough functional check. It means that the test designed to determine the required level of additional protection (see clause 2.4) should be followed by a functional check initiated after each level of HEMP impact. Clearly, this makes the test set much more complex, as after each higher amplitude pulse, the EUT should undergo the functional check. The EUT should be reconnected to the functional check systems after each HEMP test cycle. A programmable portable test system, preprogrammed to perform the certain functional checks, can simplify the test process. Many companies manufacture such systems (DOBLE, ISA, Omicron, Megger, etc.), and they are widely used in relay protection testing.

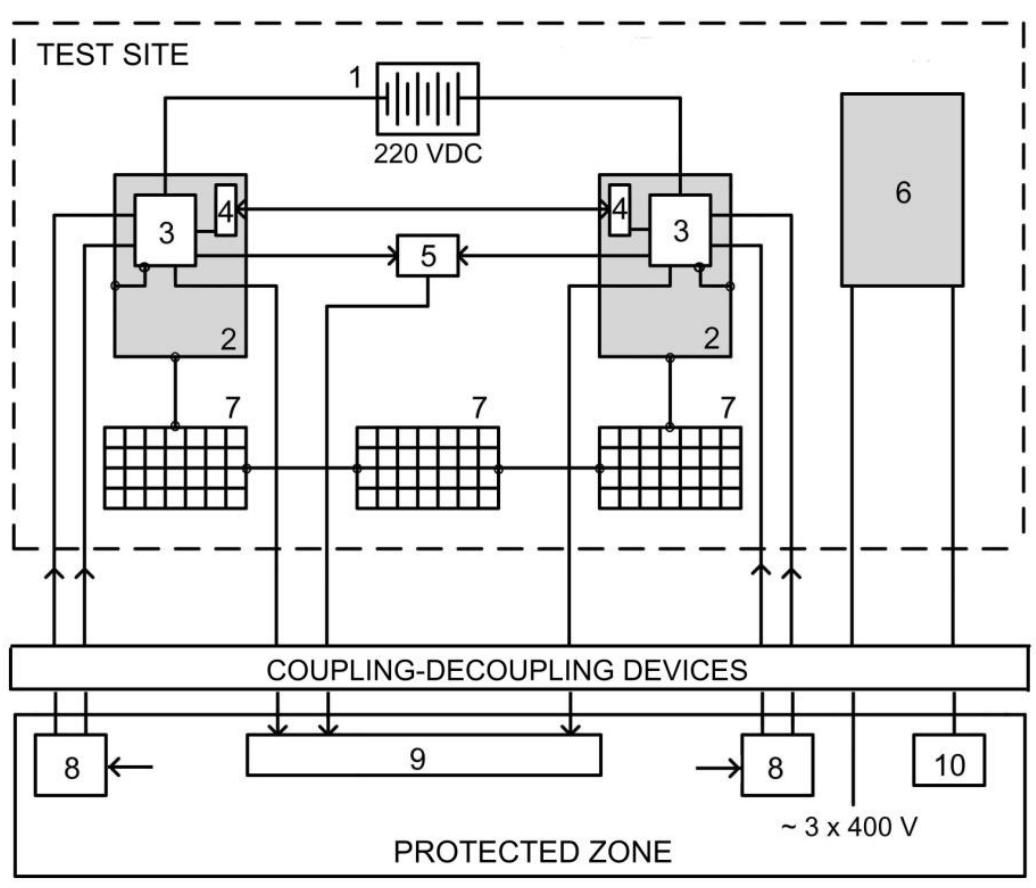

Fig2. Arrangement of EUT in the test-bed. 1 - Mobile battery 220V, 2 - Electrical cabinets distanced from one another; 3 - Tested electronics (such as Digital Protective Relays - DPR); 4 - Communication devices; 5 Lockout relay controlled via DPR output circuits; 6 - Battery charger; 7 - Set of metal meshes comprising the ground system model; 8 - simulators of different modes of EUT operation synchronized with HEMP initiation system; 9 -EUT status recorders; 10 - Load with battery charger output voltage control unit. 
Since under the HEMP affect the grounding system acts as a huge antenna absorbing electromagnetic energy over the large area, delivering it directly to the grounded electronics, the test program must include the ground system impact tests performed at two distanced EUTs connected to the same grounding system at two remote points. As the HEMP simulator is not permitted to use the mesh bonded in the concrete base as the EUT grounding system, the separate grounding system, which is realized as a sufficiently large mesh, must be provided for the test purposes. The presence of both horizontal and vertical components of the HEMP means that such a grounding mesh should be installed at $30-45^{\circ}$ to the concrete base, rather than horizontally. The mesh can be assembled from separate sections interconnected with a proper wire.

Generally speaking, power system electronic devices are connected to other electronics, sensors, power sources, electrical or electromechanical power units combined into the complex environment. For example, the relay protection system SCADA, a fire protection system, is built on such a principle. Thus, the tests should cover the complete system rather than a single unit. For relay protection systems, such an arrangement can include two cabinets each equipped with a Digital Protective Relay (DPR), the battery acting as a power source, and a battery charger. These cabinets should be spaced apart to the maximum allowable distance, connected to the grounding mesh and interconnected via a communication channel. DPR current and voltage inputs should be connected to the controlled current and voltage sources protected from the test pulse impact. To arrange such protection, the source should be located in the isolated shielded section and connected to the EUT via a special filter (coupling-decoupling circuit), allowing the transmission of signals between the EUT and the protected equipment placed in the isolated section, but preventing the ingress of the HEMP test pulse. The current and voltage source must be equipped with the remote controls to synchronize its startup with the HEMP test pulsing cycle. The state of DPR output circuits should also be considered during the test, see Fig. 2. Usually, stationary test-beds are preequipped with special shielded cables and filters designed for data transfer from the EUT, installed onto the test-bed to the isolated shielded section.

As a rule, the power system electronic equipment is installed in the metal cabinets located in all-brick or concrete buildings significantly attenuating the HEMP impact, while other devices, such as grounding system, sensors, measuring current and voltage transformers, and numerous outgoing cables, are located in an exposed area. Therefore, in real life, the different components of the common system are exposed to the electromagnetic impacts of different energy. The traditional design of the test-bed radiating antenna, Fig. 1, includes the central section, where two parallel meshes are located at a fixed distance to each other, and two side sections, where the distance between the top and the bottom meshes is decreasing. Such design allows testing different components of the system under the varied impacts of the same test pulse, since the strength of the field between the top and the bottom meshes depends heavily on the distance between them. To obtain the conditions most closely relevant to real-life situations, the components of the tested system should be located on the different sections of the test-bed.

The presence of both vertical (directed from the high-altitude detonation point to the ground surface) and large horizontal component in real-life E1 component of HEMP is another aspect which needs to be considered during the HEMP test. To allow for the consideration of both HEMP components, the elements of the tested system located in-between the top and the bottom meshes should be arranged at a certain angle to the ground surface.

The test procedure should include the registration of the EUT parameters under the HEMP with proper and adequately protected recorders. These could include external loggers, memory impulse scopes with automatic triggers, also emergency event recorders built-in the EUT and operating in parallel with other recording devices.

The selection of protective means (such as special filters, overvoltage arresters, shielded cables, etc.) or choosing an unprotected mode, should depend on the defined test objective.

\section{Test Modes and Test Pulse Parameters}

According to IEC 61000-4-25, the test for electronics immunity to HEMP must be divided into two parts: test for immunity to radiated immunity test (RI) and conducted immunity test (CI). In its turn, $\mathrm{CI}$ is divided into two types: pulse voltage applied to apparatus inputs and pulse currents induced in long cables and wires.

International Journal of Research Studies in Electrical and Electronics Engineering (IJRSEEE) Page | 26 
Determination of proper test concept (out of 6 available) is the first step for defining the rules for a certain test. Standards 61000-2-11 and 61000-5-3 describe these concepts. Concept 2b can be suitable for the EUT located inside the major reinforced concrete or all-brick building protected against lightning, but having no special protective filters. This concept allows for attenuation of EUI by $20 \mathrm{~dB}$ in the frequency band of $100 \mathrm{MHz}-30 \mathrm{MHz}$ due to the building structure. For this concept and for component E1, the strength of the radiation electrical field acting upon the tested equipment should be equal to $5 \mathrm{kV} / \mathrm{m}$ (level R4) and the strength of the magnetic field should be equal to $13.3 \mathrm{~A} / \mathrm{m}$.

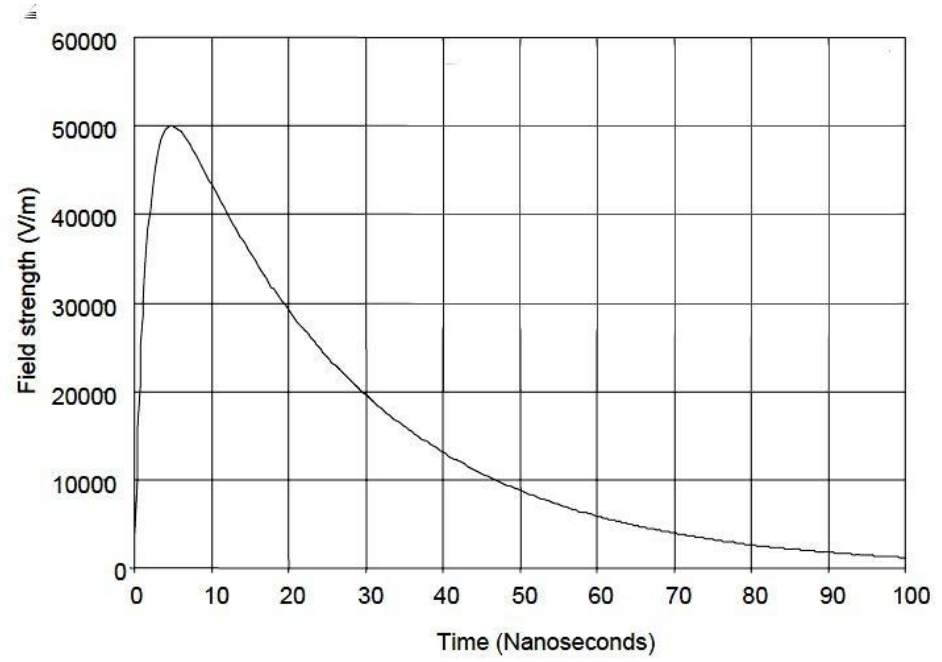

Fig3. The shape of the E1 component of HEMP according to standards: IEC 61000-2-9, IEC 61000-2-10, IEC 61000-2-11, and MIL-STD-461F.

Let us compare: for wooden buildings, providing zero EI attenuation, the strength of the electrical field equals to $50 \mathrm{kV} / \mathrm{m}$ (level R7). For the same concept and component E2, the strength of the electrical field should be equal to $10 \mathrm{~V} / \mathrm{m}$ and the strength of magnetic field $-0.08 \mathrm{~A} / \mathrm{m}$. EI pulse parameters are described in standards 61000-2-9, 61000-2-10, 61000-2-11, MIL-STD-461F: pulse rise time (front edge) is 2.5 nanoseconds, pulse width is 25 nanoseconds, pulse shape is shown in Fig. 6.

Large test-beds generating EUI with proper parameters are available in many countries. For example, there are several proper test-beds in USA (TORUS, ALECS, ARES, WSMR, ATLAS, VPBW и др.) and 3 in Russia:

- the complex ALLUR in Lenin Federal State Unitary Enterprise Russian National Electric Technical Institute, Istra, Moscow Region, see Fig. 4;

- the research center in Federal State Unitary Enterprise 12th Central Scientific-Research Institute of the Ministry of Defense of Russian Federation, Sergiyev Posad;

- the research center in 26th Central Scientific-Research Institute of the Ministry of Defense of Russian Federation, St. Petersburg.

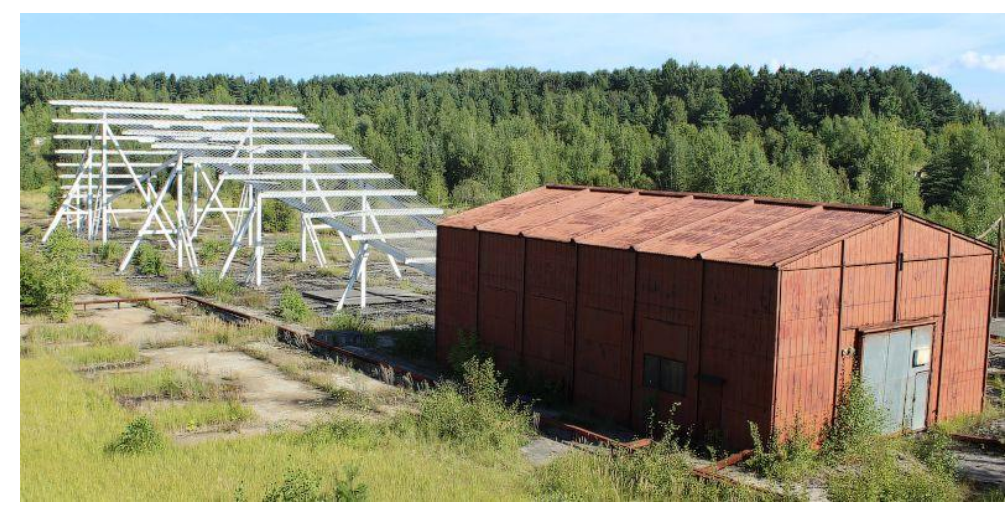

Fig4. Stationary HEMP simulator ALLUR of High-Voltage Scientific-Research Center of Federal State Unitary Enterprise Russian National Electric Technical Institute (Istra, Moscow Region). Overall dimensions: 100m x $35 \mathrm{~m} \times 13.5 \mathrm{~m}$; displacement volume: $10 \mathrm{~m} \times 10 \mathrm{~m} \times 10 \mathrm{~m}$; pulse shape: $2.5 / 25$ nanoseconds; maximum strength of electric field pulse: $70 \mathrm{kV} / \mathrm{m}$. 
Such simulators are also available in France, Germany, Sweden, Switzerland, Italy, Israel, Netherlands, Czech Republic, Poland, Ukraine, China, and Japan.

The next step is to choose the CI test level according to IEC 61000-4-25. For the concept $2 b$, due to the presence of cables connected to the tested facility and not buried in the ground, the level of test impact should correspond to E8 (to allow for 50\% probability of the facility immunity) or E9 (to allow for $99 \%$ probability). For E8 level, it is assumed that the tested facility is immune to the pulse voltage of $8 \mathrm{kV}$, and for $\mathrm{E} 9$ level - to the pulse voltage of $16 \mathrm{kV}$. A probability of $50 \%$ is deemed as normal according to the standard and can be applied to the civil equipment.

By "CI test voltage pulse" the so-called "Electrical Fast Transient (EFT) pulse" with parameters is meant (except the test voltage amplitude), and the test procedure is well described in IEC 61000-4-4, see Fig. 5.

The amplitude of the HEMP test voltage (designated as "special") is marked as " $\mathrm{X}$ " in Table 1 of this standard and corresponds to E8 and E9 levels.

Previously, EFT generators with a required level of output voltage $8 \mathrm{kV}$ were manufactured by TESEQ, Kentech Instruments Ltd. and Thermo Electron Corp. (see Table 1), and were built on triggered vacuum spark gaps generating test pulses. With the advent of solid-state switching elements such as IGBT-transistors, triggered vacuum spark gap generators were obsolete by all three companies, as the pulses generated by the transistors were much more stable and "correct" compared to pulses generated by the vacuum spark gaps. Unfortunately, improved stability of generated pulses was accompanied by the decrease of their amplitude.

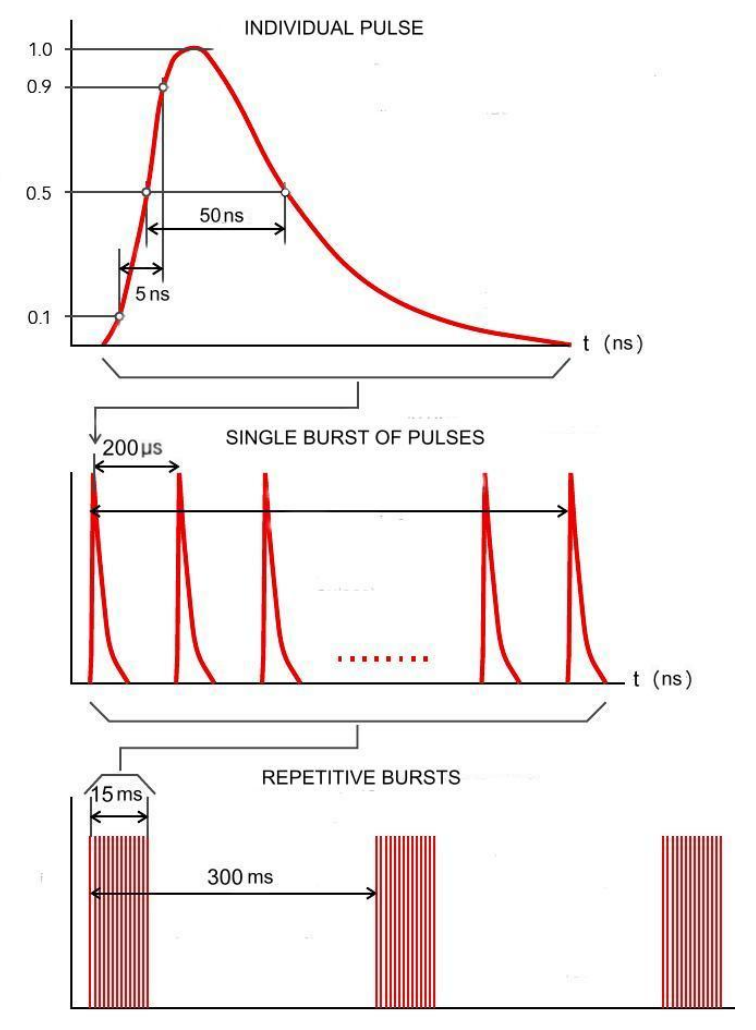

Fig5. Electrical Fast Transient (EFT) - fast pulse (IEC 61000-4-4).

Table1. The maximum amplitude of 5/50 nanoseconds output voltage pulse of EFT generators (IEC 61000-4-4) available in the market.

\begin{tabular}{|l|l|l|}
\hline Type of EFT generator & Manufacturer & Max. amplitude of output voltage pulses, $\mathrm{kV}$ \\
\hline PEFT 8010 & Haefely EMC Technology & 7.3 \\
\hline NSG 2025* & TESEQ & 8 \\
\hline J0101031/3* & Kentech Instruments Ltd. & 8 \\
\hline KeyTek ECAT E421* & Thermo Electron Corp. & 8 \\
\hline FNS-AX3-A16B & NoiseKen Laboratory Co. & 4.8 \\
\hline EFT 500N8 & EMTEST & 7 \\
\hline
\end{tabular}


Main Principles of Electromagnetic Pulse Immunity Test Methods for Power System Electronics

\begin{tabular}{|l|l|l|}
\hline TRA3000 & EMC Partner & 5 \\
\hline EFT 6501 & Schaffner & 4.4 \\
\hline EFT-4060B & Shanghai Yi PaiElectronmagneticTechn. & 6.6 \\
\hline EFT500 & Suzhou 3Ctest Electronic Co. & 5 \\
\hline AXOS8 & Hipotronics & 5 \\
\hline
\end{tabular}

obsolete* $^{*}$

My analysis shows that EFT generators presently available in the market do not fully satisfy the requirements of pulse amplitude standards $(8 \mathrm{kV})$. Generator type PEFT 8010 manufactured by a Swedish company, Haefely EMC Technology, best matches the required pulse amplitude value.

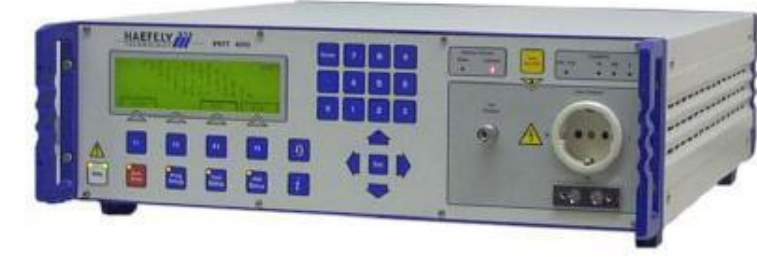

a)

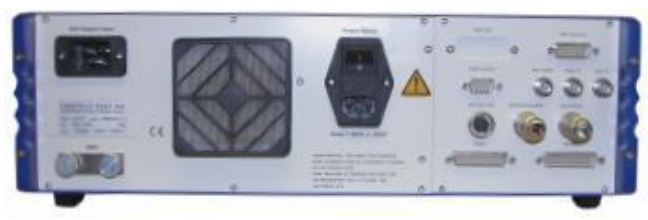

b)

Fig6. EFT generator type PEFT 8010 with the maximum pulse amplitude of $7.3 \mathrm{kV}$, manufactured by Haefely EMC Technology (Sweden), a-front panel view, в-rear panel view.

\section{Performance Criteria}

The acceptable type of certain EUT response to electromagnetic interferences during and after the certain test type is known as the performance criteria. Acceptable responses are listed below:

- Graphical distortions on EUT display, display flickers or blinks off.

- Display is showing incorrect data.

- Signals or data distortion or loss.

- Communication channel distortion or total loss.

- Sensor malfunctions.

- System false activation.

- Sharp degradation of system's ability to handle and transmit the information, and maloperation of the system.

- Software failures.

- System hangs.

- Automatic restart of computerizing electronic system.

- Complete system failure due to the power source fault or fuse blowing in feed circuits.

- Physical damage to the internal electronic components of EUT.

Basic standard IEC 61000-4-25 [4] (Par. 9) describes only 5 types of performance criteria summarizing the EUT responses listed above:

A) Normal performance within specified limits.

B) Temporary degradation or loss of functions or performance which is self-recoverable.

C) Temporary degradation or loss of functions or performance, which requires operator intervention or system reset. 
D) Degradation or loss-of-functions which is not recoverable, due to a loss of data or damage to equipment.

E) Degradation that can lead to a safety problem (for example fire).

For the test planning, the performance criteria should be predetermined separately for each type of test. These criteria should be used for assessing the EUT test results upon each test type. Apparently, only A and B criteria are relevant to the power system electronics, so only these two are the acceptable choices.

\section{Conclusion}

1. Due to the complexity and the high cost of the HEMP immunity test, such a test should be applied only to a limited range of equipment types considered as critically important devices, the lack of which makes even partial operation of electric energy facilities impossible.

2. The HEMP immunity test plan should start from the clear and transparent definition of the test objective or objectives.

3. The power system electronics should be tested as a whole system rather than as a set of individual devices. Such a whole system should include several electronic devices (two at least) interconnected by means of a communication channel, and connected to the common ground system, feeding source, control signal sources, etc. The plan development phase should include the system flowsheet charting and the compiling of a list of necessary equipment to perform the test.

4. Depending on the EUT type, the following should be defined in advance: 1) the list of parameters to be controlled over the HEMP impact period, 2) the parameter checkout methods, and 3) the types of apparatus needed to record the changes of the parameters during the test performance.

5. The results of the HEMP impact can become evident only after a certain period following the test. Thus, the control of the EUT state during the test should be supplemented by the full EUT functional check performed after the test on the HEMP simulator test-bed and then after feeding a high-voltage test pulse to the EUT using a contact method.

6. In addition to the complete set of standard EMC tests, the test for power system equipment immunity to the HEMP should consider the following two types of impacts:

1) Electromagnetic pulse - 2 nanoseconds rise time, 25 nanoseconds pulse width and 5 to $50 \mathrm{kV} / \mathrm{m}$ field strength.

2) Fast pulse (EFT) fed to EUT inputs using contact methods - 5/50 nanoseconds, $8 \mathrm{kV}$ amplitude.

7. Standard criteria A and B should be selected as a performance criterion for power system electronics.

\section{REFERENCES}

[1] Gurevich V. Cyber and Electromagnetic Threats in Modern Relay Protection. - Taylor \& Francis Group, Boca Raton, 2015, 205 p.

[2] Gurevich V. Protection of Substation Critical Equipment against Intentional Electromagnetic Threats. - Willey, 2016, $300 \mathrm{p}$.

[3] Gurevich V. I. EMP and Its Impact on Electrical Power System: Standards and Reports. Problems of Power Engineering, 2016.

[4] IEC 61000-4-25 Electromagnetic compatibility (EMC) - Part 4-25: Testing and measurement techniques - HEMP immunity test methods for equipment and systems.

[5] MIL-STD-461F Requirements for the Control of Electromagnetic Interference Characteristics of Subsystems and Equipment, RS105, 2007.

[6] Gurevich V. I. Problems in Testing Digital Protective Relays for Immunity to Intentional Destructive Electromagnetic Impacts. Continuation of the Theme. Electrical Engineering \& Electromechanics, 2015, No. 6, pp. 66-69. 


\section{AUTHOR's BIOGRAPHY}

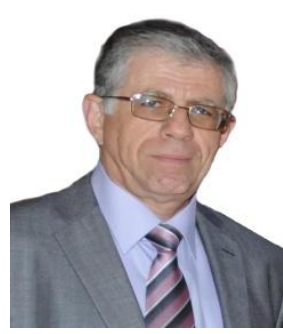

Vladimir I. Gurevich was born in Kharkov, Ukraine, in 1956. He received an M.S.E.E. degree (1978) at the Kharkov Technical University, named after P. Vasilenko, and a Ph.D. degree (1986) at Kharkov National Polytechnic University.

His employment experience includes: teacher, assistant professor and associate professor at Kharkov Technical University, and chief engineer and director of Inventor, Ltd.

In 1994, he arrived in Israel and works today at Israel Electric Corp. as a Senior specialist and Head of section of the Central Electric Laboratory.

He is the author of more than 200 professional papers and 13 books and holder of nearly 120 patents in the field of electrical engineering and power electronics. In 2006 he was Honorable Professor with the Kharkov Technical University. 